\title{
Salt, Cold, and Drought Stress on Einkorn and Bread Wheat during Germination
}

\author{
Nusret Zencirci (D)*,1, Hakan Ulukan ${ }^{(D)}$, Bülent Ordu ${ }^{(D)}$, Didem Aslan ${ }^{(D)}$, \\ Hakan Tahiri Mutlu $\stackrel{(D)}{3}$, Mehmet Örgeç ${ }^{(D)}$
}

\footnotetext{
${ }^{1}$ Bolu Abant İzzet Baysal Univ., Sciences and Art Faculty, Biology Dept., 14200, Gölköy, Bolu, Turkey

${ }^{2}$ Ankara Univ., Agricultural Faculty, Field Crops Dept., Dışkapı, Ankara, Turkey

${ }^{3}$ Bolu Abant İzzet Baysal Univ., Economics and Administrative Sciences Faculty, Business Admin. Dept., 14200, Gölköy, Bolu, Turkey
}

\begin{abstract}
Climate changes prompt salt, cold, and drought stresses especially during early crop growth stages. The damages during germination in wheat may even destroy whole crop. Here, 12 bread and 10 einkorn wheats entries were distressed under salt, cold and drought. Germination rate and germination power, coleoptile, shoot length, root length, shoot to root length ratio, root fresh and dry weight and root fresh to dry weight ratio were quantified under six salt, cold, drought stresses and one control. After ANOVA and LSD discriminated the entries, stress tolerance indices differentiated six tolerant and six susceptible entries. MANOVA, Pillai's Trace and Wilks' Lambda tests finalized the stress testing. Shoot and root length, root fresh and dry weight highly differed under salt, cold and drought. Bayraktar-2000 well tolerated salt, drought, salt-drought and salt-cold-drought; Gerek-79 salt, salt-drought, salt-cold-drought; Momtchil salt, cold and salt-cold-drought; İkizce-96 salt, drought and salt-drought. Einkorn Population 14 was susceptible to all stresses except cold and salt-cold; Population 15 to salt, salt-drought and salt-cold-drought; Population 11 to salt, drought, saltdrought and salt-cold-drought. These stresses sharply decreased shoot and root length, root fresh and root dry weight. The higher \% decreases under salt, cold and drought were in shoot $(59.72,63.25$ and 23.17$)$ and root length $(32.91,51.77$ and 34.69), root fresh $(44.32,49.11$ and 38.88) and root dry weight $(21.63,42.14$ and 41.97). Moreover, Pillai's trace and Wilks' Lambda tests differentiated both characters and entries $(\mathrm{P}<0.01)$. In conclusion, Momtchil, Gerek-79, Bayraktar2000, Populations 5, 6, and 1 are well endorsed against triple seedling stresses.
\end{abstract}

\section{ARTICLE HISTORY}

Received: January 03, 2019

Revised: March 16, 2019

Accepted: March 21, 2019

\section{KEYWORDS}

Bread wheat (Triticum

aestivum L.),

Einkorn (T. monococcum ssp. monococcum),

Cold,

Drought,

Salt

\section{INTRODUCTION}

Wheat has been the main source of food in central - west Asia and Mediterranean basin since the beginning of agriculture [1-4]. It has been cultivated for more than ten thousand years and kept providing staple nutrition for humans since then. Today's global wheat production of

*CONTACT: Nusret ZENCIRCI $\bigotimes$ nzencirci@yahoo.com Bolu Abant İzzet Baysal Univ., Sciences and Art Faculty, Biology Dept., 14200, Gölköy, Bolu, Turkey 
about 670.8 million tons per year directly influences human survival and life quality by leading the production of various foods, including bread, pasta, noodles, cakes, and biscuits.

Einkorn (T. monococcum ssp. monococcum L.), the first primary cultivated wheat ancestor emerged around the Karacadağ Mountains in the southeastern Turkey [5]. It has survived as an animal feed [6] in Turkey, Italy, and Bulgaria [2] and has become a popular human food today because of its health supporting characteristics [7].

Stresses like salt, cold or drought applies a force on a unit wheat area as well [8] and restricts its growth and decreases yield [9-12]. Many abiotic factors may affect wheat, reduce productivity and produce stress responses, $31.56 \%$ by heat, $26.61 \%$ by drought, and $23.38 \%$ by salt. Salinity, drought or cold adversely worsens crop yield and quality, limits water absorption, decreases soil osmotic potential (Izadi et al., 2014), induces water deficit, and causes morphological, physiological and biochemical deteriorations, and finally, restricts yield. The salt stress, for example, affects the wheat crop 20-30\% [13, 14], cold [15] and drought $100 \%$ [16] across the world.

Characterizing genetic resources with efficient screening tests, under in vivo and / or in vitro salt, cold and drought stresses during germination may identify new salt, cold and drought resistant genotypes [17]. Many studies on single or double biotic or abiotic stresses have been carried out [18-20] but not more than two biotic or abiotic stresses have not yet been fully illuminated. Because combined stresses involve numerous complex physiological, molecular and cellular factors and cause alterations in several plant processes [21].

Statistical analysis for complex characters like we had here require some detailed statistical methods involved. Analysis of variance (ANOVA) was followed by the least significant difference (LSD) comparison (25) in order to test wheat genotypes against salt, cold, and drought in a factorial restricted randomized block design which may serve as the first step in the stress testing procedures (24). Secondly, stress tolerance indices based on the cultivar rankings effectively differentiate tolerant and susceptible entries (24-27). Lastly, multivariate analysis of variance (MANOVA), Wilks' Lambda test, and Pillai's Trace tests, based on the most and the least deteriorated cultivars as well as the most degraded germination characters, comprehensively complete the stress testing through the sample means (26).

All of these abovementioned methods were, in this study, applied during the germination stage in order to investigate germination rate, germination power, coleoptile length, shoot length, root length, shoot to root length ratio, root fresh weight, root dry weight, and root fresh to dry weight ratio of 12 bread and 10 einkorn wheat entries under seven levels of salt, cold and drought stresses.

\section{MATERIAL and METHODS}

\subsection{Plant material}

The plant material was 12 bread (Triticum aestivum L.) and 10 einkorn wheats (Triticum monococcum ssp. monococcum) entries. Bread wheat cultivars grown in various wheat growing regions in Turkey were Gerek-79, İkizce-96, Kıraç-66, Kenanbey, Flamura-85, Momtchil, Bayraktar-2000, Tosunbey, Pandas, Pehlivan, Demir-2000 and Gün-91. Einkorn populations from different einkorn growing regions were 1, 2, 4, 5, 6, 9, 10, 11, 14, and 15 (Table 1). The bread wheat cultivar seeds were provided by various agricultural research institutes in Turkey while einkorn population seeds were collected from western Black Sea Region, Turkey (Table $1)$. 
Table 1. Twelve bread and ten einkorn wheat entries tested against seven levels of salt, cold, and drought stresses.

\begin{tabular}{|c|c|c|c|}
\hline Numbers & Entry & Origin & $\begin{array}{c}\text { Stress resistance/ } \\
\text { tolerance }\end{array}$ \\
\hline 1 & Gerek-79* & ${ }^{2}$ TARI & $\mathrm{D} / \mathrm{S} / \mathrm{C}$ \\
\hline 2 & İkizce-96 & ${ }^{1} \mathrm{CRIFC}$ & $\mathrm{C}$ \\
\hline 3 & Kıraç-66 & ${ }^{2}$ TARI & $\mathrm{D} / \mathrm{S} / \mathrm{C}$ \\
\hline 4 & Kenanbey & ${ }^{1} \mathrm{CRIFC}$ & $\mathrm{C}$ \\
\hline 5 & Flamura-85 & ${ }^{3}$ Thrace ARI & $\mathrm{S}$ \\
\hline 6 & Momtchil & ${ }^{3}$ Thrace ARI & $\mathrm{S}$ \\
\hline 7 & Bayraktar-2000 & ${ }^{1}$ CRIFC & $\mathrm{D} / \mathrm{S} / \mathrm{C}$ \\
\hline 8 & Tosunbey & ${ }^{1} \mathrm{CRIFC}$ & $\mathrm{C}$ \\
\hline 9 & Pandas & ${ }^{4}$ EMARI & $\mathrm{S}$ \\
\hline 10 & Pehlivan & ${ }^{3}$ Thrace ARI & $\mathrm{S}$ \\
\hline 11 & Demir-2000 & ${ }^{1}$ CRIFC & $\mathrm{D} / \mathrm{S} / \mathrm{C}$ \\
\hline 12 & Gün-91 & ${ }^{1} \mathrm{CRIFC}$ & $\mathrm{D} / \mathrm{S} / \mathrm{C}$ \\
\hline 13 & Population 1 & Bolu, Seben, Haccağız Village & \\
\hline 14 & Population 2 & Bolu, Seben, Boğaz Region & \\
\hline 15 & Population 4 & Bolu, Seben, Kavaklı Yazı Village, Field \# 1 & \\
\hline 16 & Population 5 & Bolu, Seben, Kavaklı Yazı Village, Field \# 2 & \\
\hline 17 & Population 6 & Bolu, Seben, Kavaklı Yazı Village, Field \# 3 & \\
\hline 18 & Population 9 & Kastamanonu, İhsangazi, Çatalyazı Village & \\
\hline 19 & Population 10 & Kastamanonu, İhsangazi, Uzunoğlu District & \\
\hline 20 & Population 11 & Kastamanonu, İhsangazi, Çay District & \\
\hline 21 & Population 14 & Kastamanonu, İhsangazi, Center & \\
\hline 22 & Population 15 & Kastamanonu, İhsangazi, Center & \\
\hline \multicolumn{4}{|c|}{$\begin{array}{l}{ }^{1} \text { CRIFC : Central Research Institute for Agricultural Research, Ankara, } \\
{ }^{2} \text { TARI : Transitional Zone Agricultural Research Institute, Eskişehir, } \\
{ }^{3} \text { Thrace ARI : Thrace Agricultural Research Institute, Edirne, } \\
{ }^{4} \text { EMARI : East Meditteranean Agricultural Research Institute, Adana. } \\
\text { * Growth type of bread wheat cultivars; W: Winter; W/F: Winter / Facultative; S: Spring. D: Drought; S: Salt; C: } \\
\text { Cold. }\end{array}$} \\
\hline
\end{tabular}

\subsection{Sterilization}

Seeds in all salt, cold and drought tests were first surface-sterilized 30 seconds in $96 \%$ ethanol, 15 minutes in $10 \% \mathrm{NaClO}$, and rinsed twice in distilled $\mathrm{H}_{2} \mathrm{O}$ [22].

\subsection{Stress tests}

\subsubsection{Salt stress}

Ten seeds in each of three replicates for a given wheat entry were germinated on Whatman number 1 wet filter paper under seven salt $(\mathrm{NaCl})$ levels. The levels were $5 \mathrm{ml}$ doses of 0 (control), $0.05 \mathrm{M}, 0.10 \mathrm{M}, 0.15 \mathrm{M}, 0.20 \mathrm{M} 0.25 \mathrm{M}$ and $0.30 \mathrm{M}$ of salt. $\mathrm{pH}$ in each petri dish was kept at $5.9 \pm 1[22]$.

\subsubsection{Cold stress}

Three replicates by ten seeds in each wheat entry were germinated on Whatman number 1 wet filter paper under seven cold stress levels of $2,0,-2,-4,-6$ and $-8{ }^{\circ} \mathrm{C}$, and the control $\left(23 \pm 1^{\circ} \mathrm{C}\right)$. $\mathrm{pH}$ was $5.9 \pm 1$ during the tests [22]. 


\subsubsection{Drought stress}

Ten seeds in all three replicates of each wheat entry were germinated on Whatman number 1 under seven levels of drought stress induced by PEG 6000 of $0.09 \mathrm{M}, 0.17 \mathrm{M}, 0.25$ $\mathrm{M}, 0.34 \mathrm{M}, 0.43 \mathrm{M}, 0.51 \mathrm{M}$ and $0.00 \mathrm{M}$. The $\mathrm{pH}$ value was fixed at $5.9 \pm 1$ [22] during the experiment.

\subsection{Germination Tests}

The seeds were germinated at $23 \pm 1{ }^{\circ} \mathrm{C}$ for 8 days (ISTA 2017) and tested in vitro under salt, cold, and drought. Each abovementioned stress consisted of seven stress levels. Germination rate, germination power, coleoptile length, shoot length, root length, shoot to root length ratio, root fresh weight, root dry weight, and root fresh to dry weight ratio were measured [23].

\subsection{Statistical Analysis}

The experiments were set up in a three-replicate (blocks) randomized complete block design (RCBD) with each stress in a factorial restriction [24]. Firstly, Fisher's protected test (F) and the mean separation by the least significant difference [LSD; 25] followed the analyses of variance (ANOVA), which were run in SPSS 21. Secondly, cultivar rank based stress tolerance indices, which were calculated in EXCEL differentiated six tolerant (Gerek-79, Gün-91, İkizce96, Bayraktar-2000, Pehlivan, and Momtchil; approximately tolerant $25 \%$ of all entries) and six susceptible (Population 1, Population-2, Population 4, Population-5, Population 6, and Population 14; approximately susceptible $25 \%$ of all entries) wheat entries [24, 26-28] Finally, the sample mean of four the least (Gerek-79, İkizce-96, Bayraktar-2000, and Pehlivan) and the most deteriorated (Population 1, Population 4, Population 6, Population 14) wheat entries and germination characters (shoot length, root length, root fresh weight, and root dry weight) were utilized to end up the multivariate analysis of variance (MANOVA), Pillai's Trace, and Wilks' Lambda tests [26].

\section{RESULTS and DISCUSSION}

Abiotic stresses induced by environmental factors cause serious damages on crop plants including wheat. One or two-way stress studies have mostly been carried out in the literature up to now. In nature, however, stresses influence crop plants in somehow more than two-way combined manners as it we had tested here in this study. Germination is one of the most stress vulnerable growth stages of the crops. Biotic or abiotic factors during germination are so critical since they worsen crop establishment and reduce yield. Salt, drought and cold are the most devastating biotic factors, especially on salt sensitive plants during germination and early seedling stages. The higher the salt concentration in the soil occurs the lower the plants germinates (29). The decreased water intake by osmotic limitations under salt, cold and drought stresses prevents the germination (30).

Here, the most stress destructed germination characters shoot length, root length, root fresh weight, and root dry weight were presented but not the other least degraded ones i.e. germination rate, germination power, coleoptile length, shoot to root length ratio, and root fresh to dry weight ratio. Blocking was effective (Table 2) in this factorially restricted randomized complete block design except for root dry weight under salt and cold stresses. All salt, cold, and drought stress types highly differentiated $(\mathrm{p}<0.01)$. Similarly, wheat entries did highly $(\mathrm{p}$ $<0.01)$ or just significantly $(\mathrm{p}<0.05)$ except root fresh weight under cold and drought. Likewise, stress levels under all three stresses differentiated highly $(\mathrm{p}<0.01)$ or just significantly $(\mathrm{p}<0.05)$. No stress type by wheat entry interaction was determined. 
Table 2. Fisher's protected $F$ value for shoot length, root length, root fresh weight, and root dry weight.

\begin{tabular}{|c|c|c|c|c|c|c|c|c|c|c|c|c|c|}
\hline & & \multicolumn{3}{|c|}{ Shoot length $(\mathrm{cm})$} & \multicolumn{3}{|c|}{ Root length $(\mathrm{cm})$} & \multicolumn{3}{c|}{ Root fresh weight (mg) } & \multicolumn{2}{c|}{ Root dry weight (mg) } \\
\hline $\begin{array}{c}\text { Sources } \\
\text { of variation }\end{array}$ & D.F. & Salt & Cold & Drought & Salt & Cold & Drought & Salt & Cold & Drought & Salt & Cold & Drought \\
\hline Blocks & 2 & $6.75^{* *}$ & $12.88^{*}$ & $8.64^{*}$ & $8.87^{* *}$ & $4.19^{* *}$ & $3.34^{*}$ & $4.08^{*}$ & $4.27^{* *}$ & $9.02^{* *}$ & $0.42^{\text {ns }}$ & $3.02^{\text {ns }}$ & $2.97^{\text {ns }}$ \\
\hline Stress types & 153 & $27.50^{* *}$ & $92.16^{* *}$ & $56.25^{* *}$ & $17.26^{* *}$ & $24.69^{* *}$ & $44.18^{* *}$ & $22.88^{* *}$ & $20.86^{* *}$ & $56.16^{* *}$ & $18.15^{* *}$ & $17.34^{* *}$ & $39.53^{* *}$ \\
\hline Entries & 21 & $2.21^{*}$ & $28.89^{*}$ & $1.98^{*}$ & $4.00^{* *}$ & $7.16^{* *}$ & $5.91^{* *}$ & $6.91^{* *}$ & $50.72^{\text {ns }}$ & $7.68^{\text {ns }}$ & $5.22^{* *}$ & $5.47^{* *}$ & $7.11^{*}$ \\
\hline Stress Levels & 6 & $210.13^{* *}$ & $602.09^{*}$ & $456.42^{*}$ & $124.36^{* *}$ & $159.68^{* *}$ & $339.71^{* *}$ & $157.65^{* *}$ & $138.63^{* *}$ & $434.21^{* *}$ & $129.64^{* *}$ & $219.16^{* *}$ & $295.62^{* *}$ \\
\hline Stress type by entry & 44 & $0.26^{\text {ns }}$ & $1.15^{\text {ns }}$ & $0.23^{\text {ns }}$ & $0.16^{\text {ns }}$ & $0.45^{\text {ns }}$ & $0.25^{\text {ns }}$ & $0.20^{\text {s }}$ & $0.34^{\text {ns }}$ & $0.27^{\text {ns }}$ & $0.10^{\text {ns }}$ & $0.65^{\text {ns }}$ & $0.25^{\text {ns }}$ \\
\hline Error & 922 & & & & & & & & & & & & \\
\hline
\end{tabular}

D.F.: Degrees of freedom; ${ }^{*}$ statistically significant at $p>0.05 ;{ }^{* *}$ statistically significant at $p>0.05$; ns: non-significant. 


\subsection{Stress tolerance indices of genotypes under three stress}

Stress rank indices differentiated bread and einkorn wheat entries under individual or multi-stresses. Approximately, $25 \%$ of all 22 wheat entries were determined tolerant and $25 \%$ susceptible under each stress alone or in two-way or three-way combinations [27, 28]. Bread wheat cultivars Bayraktar-2000, Gerek-79, İkizce-96, Demir-2000, Gün-91, Momtchil, and Flamura-85 and einkorn wheat populations 1, 6, 4, 2, 5, and 9 behaved tolerant under various stress combinations. Bread wheat cultivars Momtchil, Gerek-79, Bayraktar-2000 and einkorn populations 5, 6, 1 were the tolerant wheat entries where salt, cold and drought stresses evaluated together (Table 3 ).

Bread wheat and einkorn entries differed against salt, cold, and drought stresses alone or in combinations. These were einkorn populations 9, 10, 11, 14, 15 and bread wheat cultivars Demir-2000, Pehlivan, İkizce-96, Kıraç-66, Tosunbey, and Bayraktar-2000. Under three stresses, einkorn populations 10,11,14, 15 and bread wheat cultivars Tosunbey and Kıraç-66 were susceptible to salt-cold-drought. When two or three stresses were evaluated together Population 10 was the only genotype susceptible to salt-drought and salt-cold-drought. Kiraç66 was susceptible under the combinations of salt-cold, salt-drought and cold-drought, with stress tolerance indices of $17.17,14.06$, and 14.48, respectively. Population 10 was the most susceptible to salt (20.56), drought (17.11), cold-drought (18.94), and salt-cold-drought (15.07), (Table 3). The most worsened characters were shoot length, root length, root fresh weight, and root dry weight under salt, cold, and drought. 
Table 3. Six tolerant and six susceptible bread and einkorn wheat entries selected by their stress tolerance rank indices under one-, two-, and three-way salt, cold, and drought stresses.

\begin{tabular}{|c|c|c|c|c|c|c|c|}
\hline \multirow{2}{*}{$\begin{array}{c}\text { Tolerance } \\
\text { / Susceptibility }\end{array}$} & \multicolumn{7}{|c|}{ Stress types } \\
\hline & Salt & Cold & Drought & Salt-Cold & Salt-Drought & Cold-Drought & Salt-Cold-Drought \\
\hline \multirow[t]{12}{*}{ Tolerant } & Bayraktar-2000 & Population 6 & Kenanbey & Population 6 & Bayraktar-2000 & Gerek-79 & Momtchill \\
\hline & 5.44 & 2.89 & 6.67 & 5.39 & 4.78 & 5.78 & 8.30 \\
\hline & Gerek-79 & Population 1 & Bayraktar-2000 & Population 5 & İkizce-96 & Population 6 & Gerek-79 \\
\hline & 5.44 & 5.44 & 7.33 & 6.33 & 6.17 & 6.06 & 8.48 \\
\hline & İkizce-96 & Population 4 & Gün-91 & Population 11 & Gerek-79 & Population 1 & Population 5 \\
\hline & 5.89 & 6.11 & 7.44 & 7.50 & 6.78 & 7.72 & 8.85 \\
\hline & Gün-91 & Population 2 & Momtchil & Flamura-85 & Gün-91 & Flamura-85 & Population 6 \\
\hline & 6.00 & 3.13 & 5.66 & 7.89 & 7.11 & 8.22 & 8.89 \\
\hline & Demir-2000 & Population 5 & Population 9 & Population 9 & Demir-2000 & Population 2 & Bayraktar-2000 \\
\hline & 6.78 & 6.56 & 9.00 & 8.00 & 8.00 & 8.44 & 9.44 \\
\hline & Momtchil & Momtchil & İkizce-96 & Population 4 & Kenanbey & Population 5 & Population 1 \\
\hline & 7.44 & 7.56 & 9.00 & 8.22 & 8.39 & 8.78 & 10.26 \\
\hline \multirow[t]{12}{*}{ Susceptible } & Population 4 & Demir-2000 & Pehlivan & Bayraktar-2000 & Population 9 & Pandas & Population 11 \\
\hline & 14.11 & 16.33 & 14.00 & 15.28 & 14.06 & 13.78 & 12.78 \\
\hline & Population 14 & Pehlivan & Flamura-85 & Kenanbey & Kıraç-66 & İkizce-96 & Population 15 \\
\hline & 14.56 & 16.78 & 14.00 & 15.39 & 14.06 & 14.00 & 14.26 \\
\hline & Population 15 & İkizce-96 & Population 14 & Kıraç-66 & Population 15 & Population 14 & Tosunbey \\
\hline & 17.11 & 17.78 & 14.22 & 17.17 & 15.67 & 14.17 & 14.26 \\
\hline & Population 9 & Kıraç-66 & Kıraç-66 & Pehlivan & Population 14 & Bayraktar-2000 & Kıraç-66 \\
\hline & 18.44 & 18.44 & 16.22 & 17.39 & 16.06 & 14.89 & 14.48 \\
\hline & Population 11 & Tosunbey & Population 11 & Demir-2000 & Population 11 & Pehlivan & Population 14 \\
\hline & 18.67 & 19.11 & 16.56 & 17.39 & 18.11 & 15.44 & 14.85 \\
\hline & Population 10 & Bayraktar-2000 & Population 10 & İkizce-96 & Population 10 & Demir-2000 & Population 10 \\
\hline & 20.56 & 19.22 & 17.11 & 18.22 & 18.94 & 15.67 & 15.07 \\
\hline
\end{tabular}


The shoot length, root length, root fresh weight, and root dry weight were the worst decreased germination characters. The decrease gradually occurred under salt, cold, and drought stresses (Table 4). These four characters with the least worsened Gerek-79, İkizce-96, Bayraktar-2000, and Pehlivan bread wheat cultivars and the most worsened einkorn populations $1,4,6,14$ were further chosen for multivariate analysis.

Table 4. The most stress responsive shoot length, root length, root fresh weight, and root dry weight.

\begin{tabular}{|c|c|c|c|c|c|}
\hline Stress levels & $\begin{array}{l}\text { Stress } \\
\text { Types }\end{array}$ & $\begin{array}{l}\text { Shoot length } \\
(\mathrm{cm})\end{array}$ & $\begin{array}{l}\text { Root length } \\
\text { (cm) }\end{array}$ & $\begin{array}{c}\text { Root fresh } \\
\text { weight }(\mathrm{mg})\end{array}$ & $\begin{array}{c}\text { Root dry } \\
\text { weight }(\mathrm{mg})\end{array}$ \\
\hline \multirow{3}{*}{ Control } & Salt & $12.35 \mathrm{~A}$ & $7.70 \mathrm{~A}$ & $76.24 \mathrm{~A}$ & $7.11 \mathrm{~A}$ \\
\hline & Cold & $14.94 \mathrm{~A}$ & $8.36 \mathrm{~A}$ & $77.46 \mathrm{~A}$ & $9.23 \mathrm{~A}$ \\
\hline & Drought & $14.08 \mathrm{~A}$ & $8.64 \mathrm{AB}$ & $87.26 \mathrm{~A}$ & $7.60 \mathrm{~A}-\mathrm{C}$ \\
\hline \multirow{3}{*}{ Level 1} & Salt & $10.00 \mathrm{~B}$ & $5.70 \mathrm{AB}$ & $65.88 \mathrm{AB}$ & $6.12 \mathrm{AB}$ \\
\hline & Cold & $9.72 \mathrm{~B}$ & $5.55 \mathrm{AB}$ & $52.72 \mathrm{AB}$ & $6.95 \mathrm{AB}$ \\
\hline & Drought & $12.29 \mathrm{~B}$ & $9.01 \mathrm{~A}$ & $86.41 \mathrm{AB}$ & $9.90 \mathrm{AB}$ \\
\hline \multirow{3}{*}{ Level 2} & Salt & $5.47 \mathrm{C}$ & $4.00 \mathrm{~A}-\mathrm{C}$ & $48.34 \mathrm{~A}-\mathrm{C}$ & $4.81 \mathrm{~A}-\mathrm{C}$ \\
\hline & Cold & $8.04 \mathrm{BC}$ & $4.86 \mathrm{~A}-\mathrm{C}$ & $45.31 \mathrm{~A}-\mathrm{C}$ & $4.49 \mathrm{~A}-\mathrm{C}$ \\
\hline & Drought & $7.37 \mathrm{BC}$ & 7.48A-C & $68.04 \mathrm{~A}-\mathrm{C}$ & $9.96 \mathrm{~A}$ \\
\hline \multirow{3}{*}{ Level 3} & Salt & $2.16 \mathrm{D}$ & 2.39B-D & 32.30A-D & $3.52 \mathrm{~A}-\mathrm{D}$ \\
\hline & Cold & $6.59 \mathrm{CD}$ & $4.45 \mathrm{~A}-\mathrm{D}$ & $40.67 \mathrm{~A}-\mathrm{D}$ & 3.88A-D \\
\hline & Drought & $0.74 \mathrm{D}$ & $4.49 \mathrm{~A}-\mathrm{C}$ & $36.87 \mathrm{~A}-\mathrm{C}$ & $6.73 \mathrm{~A}-\mathrm{C}$ \\
\hline \multirow{3}{*}{ Level 4} & Salt & $0.50 \mathrm{E}$ & 1.30B-E & 19.36B-E & 2.37B-E \\
\hline & Cold & $3.03 \mathrm{E}$ & $2.38 \mathrm{~B}-\mathrm{E}$ & $22.03 \mathrm{~B}-\mathrm{E}$ & $2.11 \mathrm{~B}-\mathrm{E}$ \\
\hline & Drought & $0.00 \mathrm{DE}$ & $1.85 \mathrm{C}$ & $13.78 \mathrm{C}$ & $2.94 \mathrm{~A}-\mathrm{C}$ \\
\hline \multirow{3}{*}{ Level 5} & Salt & $0.07 \mathrm{E}$ & $0.75 \mathrm{C}-\mathrm{F}$ & $12.03 \mathrm{C}-\mathrm{F}$ & $1.66 \mathrm{C}-\mathrm{F}$ \\
\hline & Cold & $1.31 \mathrm{EF}$ & $1.53 \mathrm{~B}-\mathrm{F}$ & 13.68B-F & $1.34 \mathrm{C}-\mathrm{E}$ \\
\hline & Drought & $0.00 \mathrm{DE}$ & $0.38 \mathrm{C}$ & $3.53 \mathrm{C}$ & $0.90 \mathrm{C}$ \\
\hline \multirow{3}{*}{ Level 6} & Salt & $0.00 \mathrm{E}$ & $0.00 \mathrm{E}$ & $5.92 \mathrm{C}-\mathrm{G}$ & $1.00 \mathrm{C}-\mathrm{G}$ \\
\hline & Cold & $0.04 \mathrm{FG}$ & $0.46 \mathrm{C}-\mathrm{G}$ & $4.05 \mathrm{C}-\mathrm{G}$ & $0.41 \mathrm{C}-\mathrm{G}$ \\
\hline & Drought & $0.00 \mathrm{DE}$ & $0.08 \mathrm{C}$ & $0.99 \mathrm{C}$ & $0.24 \mathrm{C}$ \\
\hline \multirow{3}{*}{$\begin{array}{c}\text { LSD and } \\
\text { CV\% }\end{array}$} & Salt & $2.35 ; 12 \%$ & $2.19 ; 19 \%$ & $10.36 ; 12 \%$ & $0.99: 13 \%$ \\
\hline & Cold & $5.22 ; 20 \%$ & $0.36 \% 8 \%$ & $24.74: 20 \%$ & $2.28 ; 24 \%$ \\
\hline & Drought & $1.79 ; 11 \%$ & 3,$31 ; 22 \%$ & $1.05 ; 11 \%$ & $0.06 ; 12 \%$ \\
\hline \multirow{3}{*}{ Decrease $(\%)$} & Salt & 100.00 & 100.00 & 92.24 & 85.94 \\
\hline & Cold & 99.73 & 94.50 & 94.77 & 95.56 \\
\hline & Drought & 100.00 & 99.07 & 98.87 & 97.60 \\
\hline
\end{tabular}

The highest $100 \%$ decrease under salt stress was in the shoot length and root length while root dry weight $(85.94 \%)$ and root fresh weight $(92.24 \%)$ decreased the least, respectively. Decrease in shoot length under cold was $99.73 \%$, in root dry weight was $95.56 \%$, in root fresh 
weight was $94.77 \%$, and in root length was $94.50 \%$. The highest decrease under cold was in shoot length (100\%), followed by root length (99.07), root fresh weight $(98.87 \%)$, and root dry weight $(97.60 \%)$, respectively. A serious decrease was observed in shoot length at level 3 under salt and drought (i.e., $0.10 \mathrm{M}$ salt and 0.17 M PEG 6000 drought). Level 6 stress $(0.25 \mathrm{M}$, $6{ }^{\circ} \mathrm{C}, 0.43 \mathrm{M}$ PEG 6000) dramatically decreased all four characters, at least up, to a percentage of $94.50 \%$ (Table 4). Further studies to evaluate the effect of stresses in combinations may help to comprehend the consequences of stresses on wheat genotypes.

\subsection{Four the best bread wheat and four the worst einkorn wheat genotypes under three stress}

Bread wheat cultivars Gerek-79, İkizce-96, Bayraktar-2000, and Pehlivan were destructed the least, tolerant in other words, under salt, cold and drought stresses. Einkorn populations 1, 4, 6 and 14 worsened the most. These eight entries were selected for multivariate analysis as mentioned below. Bread wheat cultivars had, in general, higher values for shoot length, root length, root fresh weight, and root dry weight than einkorn populations (Table 5; Figure 2).

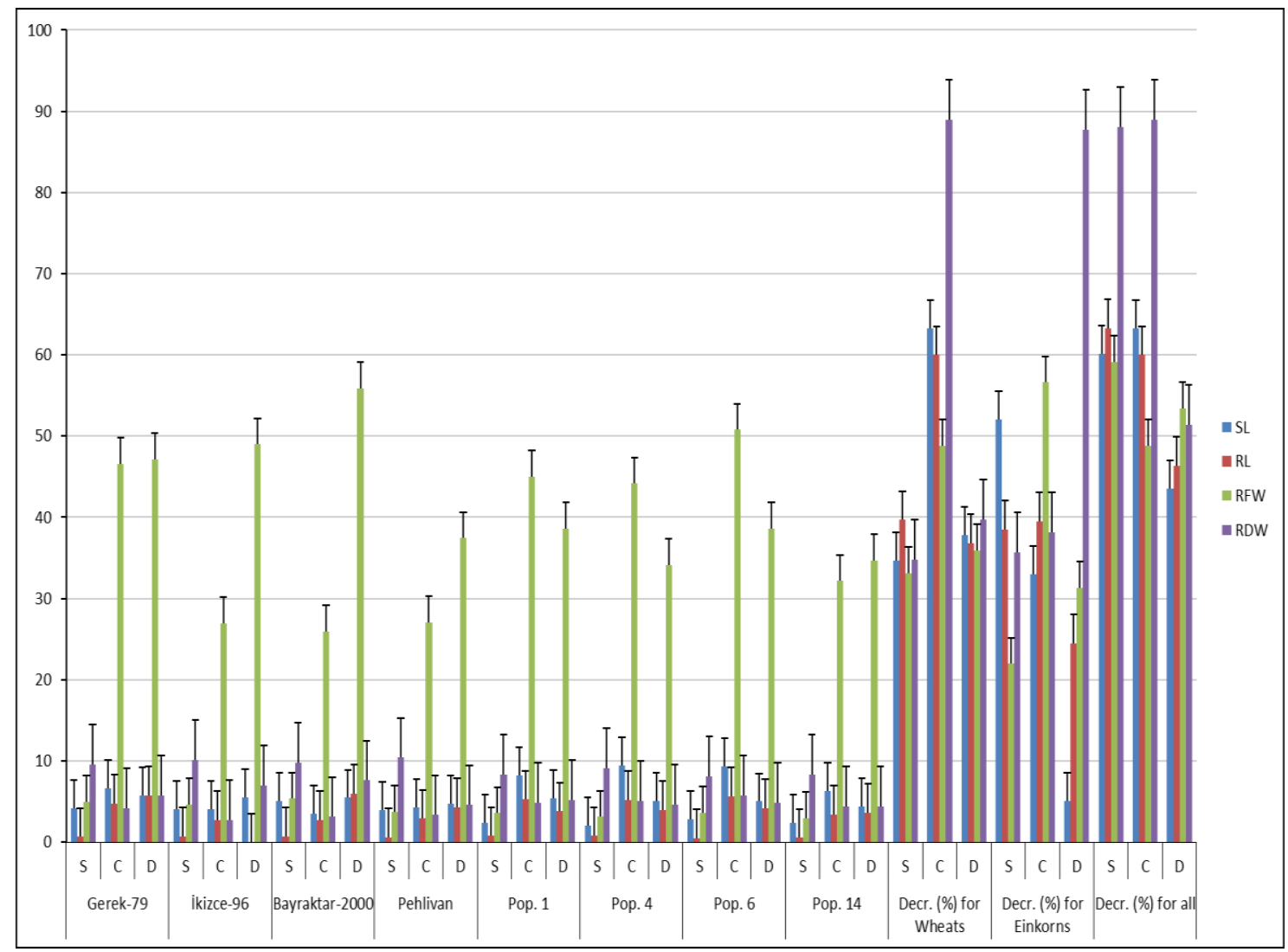

Figure 1. Four most worsened germination characters (shoot length, root length, root fresh weight, root dry weight), four einkorn populations (1, 4, 6 and 14), and four least worsened bread wheat cultivars (Gerek-79, İkizce-96, Bayraktar-2000, and Pehlivan) under salt, cold, and drought together.

Shoot length, root length, root fresh weight, and root dry weight in four bread wheat cultivars under salt stress decreased by $22.20 \%, 20.00 \%, 29.98 \%$ and $8.07 \%$, respectively. The percentage decreases in the same characters were $40.80 \%, 43.09 \%, 42.15 \%$, and $25.59 \%$, under cold and $17.42 \% ; 25.04 \%, 23.51 \%$ and $39.73 \%$, under drought stress, respectively (Table 5). The same characters were also worsened in four einkorn populations, $27.30 \%, 32.91 \%, 16.24 \%$ and $8.56 \%$, under salt stress; $32.94 \%, 39.85 \%, 36.68 \%$ and $24.19 \%$, under cold stress; and $19.23 \%, 12.41 \%, 11.65 \%$ and $15.35 \%$, under drought stress, respectively. The highest 
decreases in entries were $59.72 \%, 32.91 \%, 44.32 \%$, and $21.63 \%$ under salt; $63.25 \%, 51.77 \%$, $49.11 \%$ and $42.14 \%$ under cold stress; and $23.17 \%, 34.69 \%, 38.88 \%$ and $41.97 \%$ under drought stress, respectively (Figure 1).

Table 5. Four most worsened germination characters (shoot length, root length, root fresh weight, root dry weight), four einkorn populations (1, 4, 6 and 14), and four least worsened bread wheat cultivars (Gerek-79, İkizce-96, Bayraktar-2000, and Pehlivan) under salt, cold, and drought together, which were used in multivariate analyses.

\begin{tabular}{|c|c|c|c|c|c|}
\hline Genotypes & $\begin{array}{c}\text { Stress } \\
\text { type }\end{array}$ & $\begin{array}{l}\text { Shoot length } \\
(\mathrm{cm})\end{array}$ & $\begin{array}{l}\text { Root length } \\
\text { (cm) }\end{array}$ & $\begin{array}{l}\text { Root fresh } \\
\text { weight (mg) }\end{array}$ & $\begin{array}{c}\text { Root dry } \\
\text { weight (mg) }\end{array}$ \\
\hline \multirow{3}{*}{ Gerek-79 } & Salt & $4.20 \mathrm{ab}$ & $0.66 a-c$ & $5.00 \mathrm{ab}$ & 9.56b-1 \\
\hline & Cold & $6.69 a-k$ & $4.78 \mathrm{a}-\mathrm{g}$ & $4.65 \mathrm{a}-\mathrm{d}$ & $4.18 b-1$ \\
\hline & Drought & $5.74 a-c$ & 5.79ab & 4.71a-h & $5.79 \mathrm{c}-\mathrm{h}$ \\
\hline \multirow{3}{*}{ İkizce-96 } & Salt & $4.11 \mathrm{a}-\mathrm{c}$ & $0.75 \mathrm{a}-\mathrm{e}$ & $4.65 \mathrm{a}-\mathrm{d}$ & $10.10 \mathrm{~b}-\mathrm{h}$ \\
\hline & Cold & $4.02 \mathrm{~g}-\mathrm{s}$ & $2.71 \mathrm{j}-\mathrm{t}$ & $2.69 \mathrm{i}-\mathrm{t}$ & $2.70 \mathrm{~m}-\mathrm{u}$ \\
\hline & Drought & $5.56 \mathrm{a}-\mathrm{d}$ & $5.26 \mathrm{a}-\mathrm{I}$ & $4.89 a-g$ & $6.99 a-c$ \\
\hline \multirow{3}{*}{ Bayraktar-2000 } & Salt & $5.09 \mathrm{a}$ & $0.74 a-e$ & $5.37 \mathrm{a}$ & 9.78b-I \\
\hline & Cold & $3.48 \mathrm{~g}-\mathrm{u}$ & $2.72 \mathrm{j}-\mathrm{t}$ & $2.58 \mathrm{j}-\mathrm{u}$ & $3.11 \mathrm{~g}-\mathrm{t}$ \\
\hline & Drought & $5.47 \mathrm{a}-\mathrm{f}$ & $5.97 \mathrm{a}$ & $5.58 \mathrm{ab}$ & $7.60 \mathrm{ab}$ \\
\hline \multirow{3}{*}{ Pehlivan } & Salt & 3.96a-f & $0.60 \mathrm{a}-\mathrm{f}$ & $3.76 \mathrm{c}-1$ & 10.40a-e \\
\hline & Cold & $4.25 \mathrm{~g}-\mathrm{r}$ & $2.90 \mathrm{j}-\mathrm{r}$ & $2.70 \mathrm{i}-\mathrm{s}$ & $3.35 \mathrm{e}-\mathrm{o}$ \\
\hline & Drought & $4.74 a-0$ & $4.34 \mathrm{c}-\mathrm{k}$ & $3.74 f-p$ & $4.58 \mathrm{f}-\mathrm{q}$ \\
\hline \multirow{3}{*}{ Population 1} & Salt & $2.40 \mathrm{~h}-\mathrm{q}$ & $0.78 \mathrm{a}-1$ & $3.57 \mathrm{c}-\mathrm{n}$ & $8.30 \mathrm{i}-\mathrm{s}$ \\
\hline & Cold & $8.20 \mathrm{a}-\mathrm{e}$ & $5.28 \mathrm{a}-\mathrm{b}$ & 4.5.0a-e & $4.88 \mathrm{a}-\mathrm{d}$ \\
\hline & Drought & $5.46 \mathrm{a}-\mathrm{f}$ & $3.82 \mathrm{~h}-\mathrm{p}$ & $3.85 \mathrm{~d}-\mathrm{m}$ & $5.21 \mathrm{~d}-1$ \\
\hline \multirow{3}{*}{ Population 4} & Salt & $2.05 \mathrm{j}-\mathrm{s}$ & 0.79 ao & $3.11 \mathrm{~g}-\mathrm{p}$ & $9.11 \mathrm{~b}-\mathrm{m}$ \\
\hline & Cold & $9.47 \mathrm{a}$ & $5.17 \mathrm{a}-\mathrm{c}$ & $4.41 \mathrm{a}-\mathrm{h}$ & $5.07 \mathrm{ab}$ \\
\hline & Drought & $5.12 \mathrm{a}-\mathrm{k}$ & $3.96 \mathrm{f}-\mathrm{n}$ & 3.41h-r & $4.66 \mathrm{e}-\mathrm{p}$ \\
\hline \multirow{3}{*}{ Population 6} & Salt & $2.82 \mathrm{c}-\mathrm{n}$ & $0.53 \mathrm{a}-\mathrm{m}$ & $3.61 \mathrm{c}-\mathrm{m}$ & $8.15 \mathrm{i}-\mathrm{u}$ \\
\hline & Cold & $9.33 a-b$ & $5.62 \mathrm{a}$ & $5.07 \mathrm{a}$ & $5.79 \mathrm{a}$ \\
\hline & Drought & $5.02 \mathrm{a}-1$ & $4.19 \mathrm{e}-\mathrm{m}$ & 3.86d-e & $4.88 \mathrm{~d}-\mathrm{n}$ \\
\hline \multirow{3}{*}{ Population 14} & Salt & 2.36h-r & $0.54 \mathrm{a}-\mathrm{k}$ & $2.99 \mathrm{~h}-\mathrm{q}$ & $8.33 \mathrm{i}-\mathrm{q}$ \\
\hline & Cold & $6.35 a-1$ & $3.38 \mathrm{~g}-\mathrm{p}$ & $3.21 \mathrm{e}-\mathrm{n}$ & $4.38 \mathrm{~b}-\mathrm{g}$ \\
\hline & Drought & $4.41 \mathrm{a}-\mathrm{q}$ & $3.67 \mathrm{j}-\mathrm{s}$ & $3.46 \mathrm{~h}-\mathrm{q}$ & $4.41 \mathrm{~g}-\mathrm{r}$ \\
\hline \multirow{3}{*}{ LSD and $C V \%$} & Salt & $0.85 ; 12 \%$ & $0.06: 11 \%$ & $0.37 ; 11 \%$ & $0.84 ; 16 \%$ \\
\hline & Cold & $0.14 ; 18 \%$ & $0.09 \% 12 \%$ & $0.42 ; 14 \%$ & $0.89 \% 18 \%$ \\
\hline & Drought & $0.27 ; 9 \%$ & $0.18 ; 14 \%$ & $0.58: 15 \%$ & 0.61 \\
\hline \multirow{3}{*}{$\begin{array}{l}\text { Decrease } \\
\text { wheat (\%) }\end{array}$} & Salt & 22.20 & 20.00 & 29.98 & 8.07 \\
\hline & Cold & 40.80 & 43.09 & 42.15 & 25.59 \\
\hline & Drought & 17.42 & 25.04 & 23.51 & 39.73 \\
\hline \multirow{3}{*}{$\begin{array}{l}\text { Decrease in } \\
\text { einkorn (\%) }\end{array}$} & Salt & 27.30 & 32.91 & 16.24 & 8.56 \\
\hline & Cold & 32.94 & 39.85 & 36.68 & 24.35 \\
\hline & Drought & 19.23 & 12.41 & 11.65 & 15.35 \\
\hline \multirow{3}{*}{$\begin{array}{l}\text { Decrease } \\
\text { in all (\%) }\end{array}$} & Salt & 59.72 & 32.91 & 44.32 & 21.63 \\
\hline & Cold & 63.25 & 51.77 & 49.11 & 42.14 \\
\hline & Drought & 23.17 & 34.69 & 38.88 & 41.97 \\
\hline
\end{tabular}




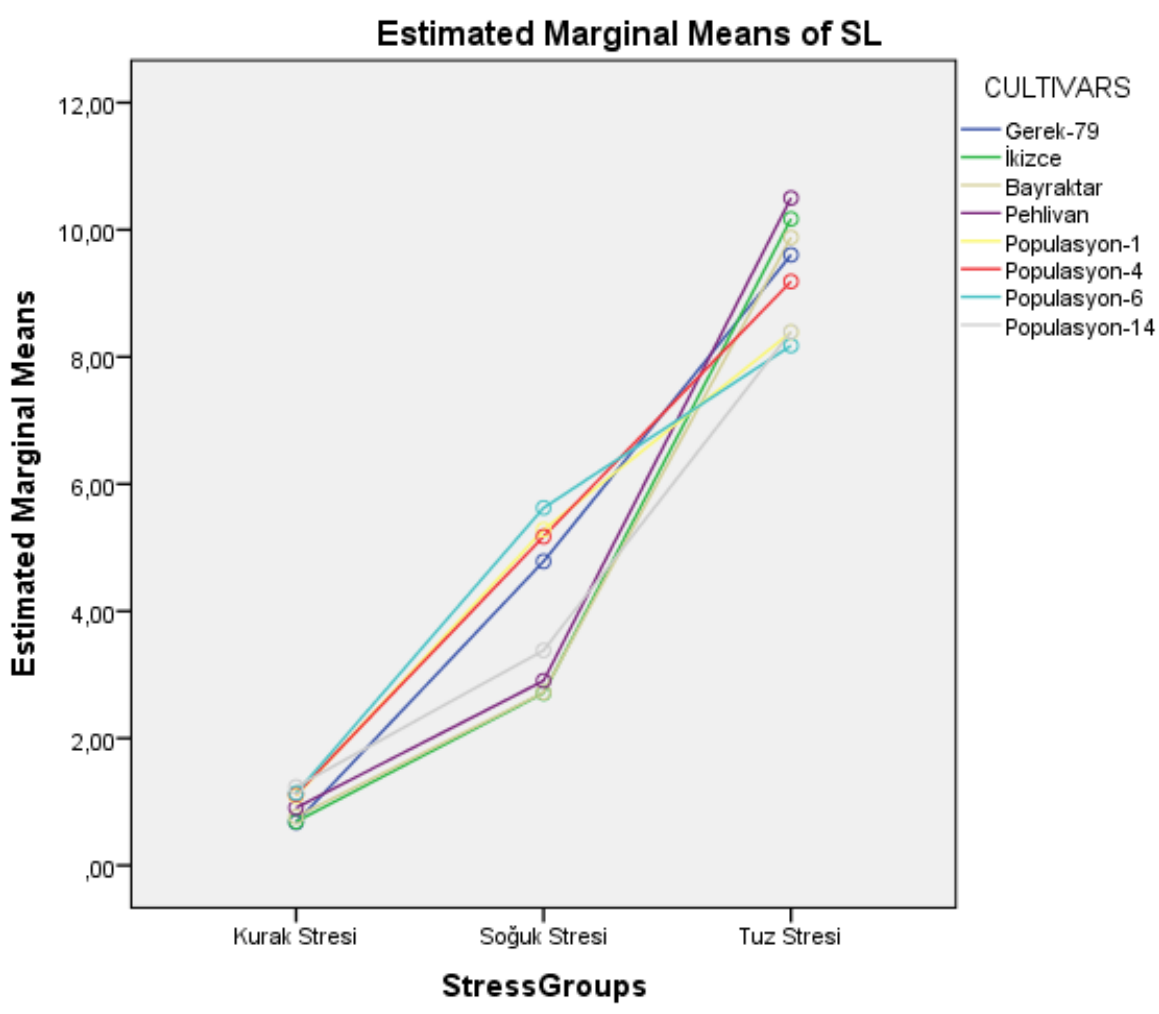

Figure 2. The shoot lengths of four most deteriorated einkorn populations (1, 4, 6 and 14), and four least worsened bread wheat cultivars (Gerek-79, İkizce-96, Bayraktar-2000 and Pehlivan) under salt, cold, and drought together, which were all three used in multivariate analyses.

\subsection{Shoot length, root length, root fresh weight, and root dry weight of the least responsive four bread wheat and the most responsive four einkorn genotypes under salt, cold, and drought}

A multivariate analysis of variance (MANOVA) was performed on germination characters, bread wheat cultivars, einkorn populations, and salt, cold, and drought stresses. Furthermore, Pillai's Trace and Wilks' Lambda tests were applied on data [26].

All salt, cold and drought stresses, wheat entries, and stress by wheat entry interaction(s) significantly $(\mathrm{P}<0.01)$ affected the independent variables (Table 7). Partial Eta squared indicated a strong effect on all three stresses and a weak effect on wheat entries and stress by wheat entry interactions. Pillai's Trace and Wilks' Lambda were highly significant $(p<0.01)$ for stress types of salt, cold, drought, wheat entries, and stress type by entry interaction. The between-subject effects were also significant for all stresses and stress by wheat entry interactions $(\mathrm{P}<0.05)$, but insignificant for wheat entries. Partial Eta squared had the highest effect on the shoot length and the lowest on the root dry weight. Stress by wheat entry interactions affected shoot length the most (Table 6). 
Table 6. MANOVA for four most worsened germination characters (shoot length, root length, root fresh weight, root dry weight), four einkorn populations (1, 4,6 and 14), and four least worsened bread wheat cultivars (Gerek-79, İkizce-96, Bayraktar-2000, and Pehlivan) under salt, cold, and drought together.

\begin{tabular}{|c|c|c|c|c|}
\hline Source & $\begin{array}{l}\text { Dependent } \\
\text { Variable }\end{array}$ & df & $\mathrm{F}$ & Partial Eta Squared \\
\hline \multirow[t]{4}{*}{ Stress types } & SL & 2 & $325.094 * *$ & 0.639 \\
\hline & $\mathrm{RL}$ & 2 & $21.512 * *$ & 0.105 \\
\hline & RDW & 2 & $10.890^{* *}$ & 0.056 \\
\hline & RFW & 2 & $42.442 * *$ & 0.187 \\
\hline \multirow[t]{4}{*}{ Cultivars } & SL & 7 & 0.571 & 0.011 \\
\hline & $\mathrm{RL}$ & 7 & 0.816 & 0.015 \\
\hline & RDW & 7 & 2.008 & 0.037 \\
\hline & RFW & 7 & 0.919 & 0.017 \\
\hline \multirow[t]{4}{*}{ Stress types* Cultivars } & SL & 14 & $3.633 * *$ & 0.121 \\
\hline & RL & 14 & $2.892^{* *}$ & 0.099 \\
\hline & RDW & 14 & $2.279 * *$ & 0.080 \\
\hline & $\overline{R F W}$ & 14 & $2.784 * *$ & .096 \\
\hline \multirow[t]{4}{*}{ Error } & SL & 368 & & \\
\hline & RL & 368 & & \\
\hline & RDW & 368 & & \\
\hline & RFW & 368 & & \\
\hline
\end{tabular}

Table 7. Pillai's trace and Wilks' lambda multivariate tests for intercept + stress types + cultivars + Stress types * cultivars.

\begin{tabular}{ll}
\hline Effect & \\
\hline Intercept & Pillai's Trace** \\
\hline Stress types & Pilks' Lambda** \\
\cline { 2 - 2 } & Willai's Trace** \\
\hline Cultivars & Pillai's Trace** \\
\cline { 2 - 2 } & Wilks' Lambda** \\
\hline Stress types * Cultivars & Pillai's Trace** \\
\cline { 2 - 2 } & Wilks' Lambda** \\
\hline
\end{tabular}

**The tests are significant at the 0.01 level.

**The mean difference is significant at the 0.01 level.

All shoot length, root length, root dry weight, and root fresh weight differed between drought, cold, and salt pairs except root dry weight and root fresh weight between cold-salt stress pairs (Table 8). Pillai's Trace and Wilks' lambda were highly significant for shoot length, root length, root dry weight under slat, cold, and drought (Table 9). 
Table 8. Multiple comparisons of shoot length, root length, root dry weight, and root fresh weight under salt, cold, and drought.

\begin{tabular}{ccccc}
\hline Dependent Variable & Stress types (I) & Stress types $(J)$ & Mean Difference $(\mathrm{I}-\mathrm{J})$ & Std. Error \\
\hline \multirow{2}{*}{ SL } & Drought & Cold & $-3.1199^{* *}$ & 0.37594 \\
\cline { 3 - 5 } & & Salt & $-8.3353^{* *}$ & 0.37594 \\
\cline { 2 - 5 } & Cold & Salt & $-5.2155^{* *}$ & 0.26583 \\
\hline \multirow{2}{*}{ RL } & Drought & Cold & $2.2706^{* *}$ & 0.45295 \\
\cline { 3 - 5 } & & Salt & $2.9703^{* *}$ & 0.45295 \\
\cline { 2 - 5 } & Cold & Salt & 0.6997 & 0.32028 \\
\hline \multirow{2}{*}{ RDW } & Drought & Cold & $1.9190^{* *}$ & 0.41178 \\
\cline { 2 - 5 } & & Salt & $1.5070^{* *}$ & 0.41178 \\
\cline { 2 - 5 } & Cold & Salt & -0.4120 & 0.29117 \\
\hline \multirow{2}{*}{ RFW } & Drought & Cold & $32.7163^{* *}$ & 3.82036 \\
\cline { 2 - 5 } & & Salt & $33.1244^{* *}$ & 3.82036 \\
\cline { 2 - 5 } & Cold & Salt & -0.4081 & 2.70140 \\
\hline
\end{tabular}

Fresh Weight. Based on observed means. The error term is Mean Square (Error) $=612,996$.

*The mean difference is significant at 0.05 level and ** at the 0.01 level.

Table 9. Pillai's trace and Wilks' lambda multivariate tests for shoot length, root length, root dry weight, and root fresh weight under salt, cold, and drought.

\begin{tabular}{lcc}
\hline & Value & $\mathrm{F}$ \\
\hline Pillai's trace & 1.451 & $241.710^{* *}$ \\
Wilks' lambda & 0.044 & $343.948^{* *}$ \\
\hline **The mean difference is significant at the 0.01 level.
\end{tabular}

Among four bread and einkorn multi-trait analyzed wheat entries, Gerek-79 and Population 14 significantly differed $(\mathrm{P}<0.05)$ for root fresh weight. Gerek-79 - Population 14 and Pehlivan - Gerek-79 highly but not significantly differed (Table 10). Pillai's trace and Wilks' lambda tests also highly significant for these cultivar pairs in shoot length, root length, root dry weight, and root fresh weight (Table 11).

Many studies about the effect of stress on wheat are conducted individually; however, biotic and/or abiotic stresses attack wheat together. Recently, some studies have been undertaken by a two- or two-way biotic-biotic, biotic-abiotic stress study approaches. Thus, it seemed appropriate to engage in this current study on the individual effects of salt, cold, and drought stresses in a combined manner on wheat.

Table 10. Multiple comparisons of shoot length, root length, root dry weight, and root fresh weight observed with bread and einkorn wheats under salt, cold, and drought.

\begin{tabular}{lccccc}
\hline $\begin{array}{l}\text { Dependent } \\
\text { Variable }\end{array}$ & CULTIVARS & CULTIVARS & $\begin{array}{c}\text { Mean } \\
\text { Difference (I-J) }\end{array}$ & Std. Error & Sig. \\
\hline RL & Gerek-79 & Populasyon 14 & 1.5549 & 0.59305 & 0.151 \\
\hline RDW & Pehlivan & Gerek-79 & -1.4550 & 0.53914 & 0.126 \\
\hline RFW & Gerek-79 & Populasyon 14 & $15.9906^{*}$ & 5.00202 & 0.032 \\
\hline
\end{tabular}

Based on observed means. 
Table 11. Pillai's trace and Wilks' lambda multivariate tests for shoot length, root length, root dry weight, and root fresh weight under salt, cold, and drought.

\begin{tabular}{lcc}
\hline & Value & $\mathrm{F}$ \\
\hline Pillai's trace & 0.143 & $1.956^{* *}$ \\
Wilks' lambda & 0.861 & $1.995^{* *}$ \\
\hline
\end{tabular}

**The mean difference is significant at the 0.01 level.

Plant germination decreases when salt concentration in the soil increases [29]. Reduced intake of water due to osmotic limitations and $\mathrm{Na}$ and $\mathrm{Cl}$ ion toxicity worsen germination characteristics [30]. Species and genotypes differ in their reactions to individual and most likely combined biotic stresses; thus, a detailed exploration is needed. Einkorn seemed more tolerant environmental stresses than bread wheats in the previous studies [19, 20, 31]. Mahmoodabad [32] reported the lowest germination rate at $2^{\circ} \mathrm{C}$, with some differences among bread wheat cultivars of Gaspard, Sardari, Cascogen, Bezostaja-1, and MV-17. Similarly, the root length was the same in Gaspard, Bezostaya-1 and Cascogen as Bezostaya 1, which has been frequently reported to have the longest shoot and root lengths at the lower temperatures. The characteristics of cultivars under different stresses differed during the germination and early seedling stages in some previous studies. Chilling temperatures between $0-12^{\circ} \mathrm{C}$ significantly delayed the onset and reduced the germination rate in the cultivated plants $[33,34]$. Cold also led to poor seed germination, uneven stand establishment, and poor crop performance [33]. Most commercial crop cultivars have been highly sensitive to cold during seed germination despite available genetic variation within and between related wild species [33, 34].

Among abiotic stresses, drought widely spreads around the world, upsetting seed germination and seedling growth, and resulting in a poor establishment and a decreased seedling growth. Poor establishment, in turn, decreases weed competitiveness, shades soil surface, restricts light interception, declines early season growth, and reduces yield. Similarly, shoot length, root length, root fresh weight, and root dry weight gradually decreased under salt, cold and drought stresses in this study (Table 4). This was parallel with the results of Mahmoodzadeh [35] who obtained significant shoot and root length differences in bread wheat cultivars. All four: shoot length, root length, root fresh weight and root dry weight characters similarly followed the same trend against salt, cold, and drought stresses in the present study.

Stress tolerance indices $[24,27,28]$ successfully differentiated bread wheat cultivars and einkorn populations under individual or combined salt, cold, and drought stresses (Table 3). Bayraktar-2000, Gerek-79, İkizce-96, Demir-2000, Gün-91, Momtchil, and Flamura-85 bread wheat cultivars and 1, 6, 4, 2, 5, and 9 einkorn wheat populations were tolerant under various stress combinations. Bread wheat cultivars Momtchil, Gerek-79, and Bayraktar-2000 and einkorn populations 5,6 , and 1 were the tolerant wheat entries when salt, cold and drought stresses evaluated together (Table 3). The values by Ali and El-Sadak [36] who compared the stress susceptibility and stress tolerance indices, mean and geometric mean productivity were not parallel with the stress indices here. When multivariate analysis variance, partial Eta squared values, and Pillai's trace and Wilks' Lambda tests were considered partial Eta revealed a strong effect for three stresses and a weak effect for wheat entries and stress by wheat interaction (Table 7) similarly to the stress tolerance indices in this study.

\section{CONCLUSION}

Previous studies have mostly investigated individual stresses and their effects on plants because of an easy testing and a statistical analysis. However, plants are simultaneously exposed to more than one stress in their real-life cycles. Therefore, studying stresses in a combined approach by multivariate analysis methods is expected to provide a better 
understanding of the stresses on wheat. This study, therefore, presented a novel approach and produced valuable results about the effects of three stresses on wheat and einkorn entries in a combined understanding.

\section{Orcid}

Nusret ZENCIRCİ https://orcid.org/0000-0003-3460-7575

Hakan ULUKAN (iD https://orcid.org/0000-0003-0203-6851

Bülent ORDU (iD https://orcid.org/0000-0002-3103-9325

Didem ASLAN (iD) https://orcid.org/0000-0001-6747-2852

Hakan Tahiri MUTLU (iD) https://orcid.org/0000-0002-8964-2696

Mehmet ÖRGEÇ (iD https://orcid.org/0000-0002-9446-7538

\section{REFERENCES}

[1] Dhanda, S.S., Sethi, G.S., Behl, R.K. (2004). Indices of Drought Tolerance in Wheat Genotypes at Early Stages of Plant Growth. J. Agron Crop Sci., 190, 6-12.

[2] Feuillet, C., Langridge, P., Waugh, R. (2007). Cereal Breeding Takes A Walk on the Wild Side. Trends Genet., 24(1), 1-32.

[3] Shahzad, A., Iqbal, M., Asif, M., Hirani, A.H., Goyal A. (2013). Growing Wheat on Saline Lands, Can A Dream Come True? Australian J. Crop Sci., 7, 515-524.

[4] Eren, H, Pekmezci, M.Y, Okay, S., Turktas, M., Inal, B., Ilhan, E., Atak, M., Erayman, M., Unver, T., Unver C.T. (2015). Hexaploid Wheat (Triticum Aestivum) Root Mirnome Analysis in Response to Salt Stress. Ann Appl Biol., 167, 2-30.

[5] Charmet, G. (2011). Wheat Domestication: Lessons for the Future. C. R. Biologies, 334, 212-220.

[6] Hidalgo, A., Brandolini, A. (2013). Nutritional Properties of Einkorn Wheat (Triticum monococcum L.). J. Sci. Food Agric., 94, 601-61.

[7] Sharma, H.C., Waines, J.G., Foster, W. (1981). Variability in Primitive and Wild Wheats for Useful Genetic Characters. Crop Sci., 21, 555-559.

[8] Kranner, I, Minibayeva, F.V., Beckett, R.P., Seal C.E., (2010). What Is Stress? Concepts, Definitions and Applications in Seed Science. Tansley Rev. New Phytol, 188, 655 - 673.

[9] Mehrotra, R., Bhalothia, P., Bansal, P., Basantani, M.K., Bharti, V., Mehrotra, S. (2014). Abscisic Acid and Abiotic Stress Tolerance-Different Tiers of Regulation. J. Plant Phys., 171,486 - 496.

[10] Pierik, R., Testerink, C. (2014). The Art of Being Flexible, How to Escape from The Shade, Salt, And Drought. J Plant Phys., 166, 5-22.

[11] Izadi, M.H., Rabbani, J., Emam, Y., Pessarakli, M., Tahmasebi, A. (2014). Effects of Salinity Stress on The Physiological Performance of Various Wheat and Barley Cultivars. J. Plant Nutr, 37, 520 - 531.

[12] Richter, J.E., Kopka, J., Zerb, C. (2015). Metabolic Contribution to Sal t Stress in Two Maize Hybrids with Contrasting Resistance. Plant Sci., 233, 107-115.

[13] Hasanuzzaman, M., Nahar, K., Mahabub, A.M.D., Bhowmik, C.P., Amzad, H.M.D., Rahman, M.M., Prasad, V., Narasimha, M., Ozturk, M., Fujita M. (2014). Potential Use of Halophytes to Remediate Saline Soils. BioMed Res. Int., 1-12.

[14] Vardar, Y., Çifci E.A. (2014). Salinity Effects on Germination Stage of Bread and Durum Wheat Cultivars. J Yuzuncu Yll Univ., 24, 127-139.

[15] Braun, H.J., Ekiz, H., Eser, V., Keser, M., Ketata, H., Marcucci, G., Morgounov, A.I., Zencirci N. (1998). Breeding Priorities of Winter Wheat Programs. In: H.-J. Braun, F. Altay, W.E. Kronstad, S.P.S. Beniwal \& A. McNab, editors. Wheat, Prospects for Global Improvement. Proc. 5th Int. Wheat Conf., Ankara, Developments in Plant Breeding, Kluwer Academic Publishers, Dordrecht, Netherlands. pp 553 - 560. 
[16] Khodabandeh, N. (2003). Cereals. Seventh Edition, Tehran University Press, pp. 78- 111. [17] Mostek, A., Börner, A., Badowiec, A., Weidner, S. (2015). Alterations in Root Proteome of Salt-Sensitive and Tolerant Barley Lines under the Salt Stress Conditions. J. Plant Phys., 174, 166-176.

[18] Karakaş, F.P. (2016). Effects of Drought and Salinity Stress on Early Seedling Growth and Antioxidant Activity in Hulled Einkorn (Triticum monococcum ssp. monococcum) and Bread (Triticum aestivum L.) Wheats. J. CRI for Field Crops, 25, 107-116.

[19] Aslan, D., Zencirci, N., Etöz, M., Ordu, B., Bataw S. (2016). Bread Wheat Responds Salt Stress Better Than Einkorn Wheat Does During Germination. Turkish J. Agric. For., 40(5), 783-794.

[20] Aslan, D., Ordu, B., Zencirci, N. (2016). Einkorn Wheat (Triticum monococcum ssp. monococcum) Tolerates Cold Stress Better than Bread Wheat (Triticum aestivum L.) During Germination. J. Field Crops Cent. Res. Inst., 25(2), 182-192.

[21] Ashraf, M., Harris P.J.C. (2013). Photosynthesis under Stressful Environments: An Overview. Photosynth, 51(2), 163-190.

[22] Baloch, M.J., Dunwell, J., Khakwani, A.A., Dennett, M., Jatoi, W.A., Channa S.A. (2012). Assessment of Wheat Cultivars for Drought Tolerance via Osmotic Stress Imposed at Early Seedling Growth Stages. J. Agric. Res., 50(3), 299 - 310.

[23] Thornley, J.M. (1998). Modelling Shoot, Root Relations, The Way Forward. Ann Bot., 81, 165-171.

[24] Zencirci, N., Eser, V., Baran, I. (1990). Comparison of Some Stability Statistics. CRIFC Publicaitions, Publication no, 2:17 (in Turkish).

[25] Gomez, K., Gomez, A.A. (1984). Statistical Procedures for Agricultural Research, 2nd edition. John Wiley and Sons: New York, USA. pp. 680

[26] Kalaycı, Ş. (2010). SPSS Uygulamalı Çok Değişkenli İstatistik Teknikleri: In: ASİL Yayın Dağıtım Ltd. Şti., Ankara, Turkey P. 116 (SPSS applied multi-variate statistic techniques. Pg 116. ASÍL Publication Casting Ltd. Co. Ankara, Turkey) [in Turkish].

[27] Askari, H., Kazemitabar, S.K., Zarrini, H.N., Saberi M.H. (2016). Salt Tolerance Assessment of Barley (Hordeum vulgare L.). Open Agric., 1, 37-44.

[28] Oyiga, B.C., Sharma, R.C., Shen, J., Baum, M., Ogbonnaya, F.C., Leon, J., Ballvora, A. (2016). Identification and Characterization of Salt Tolerance of Wheat Germplasm Using A Multivariable Screening Approach. J Agron Crop Sci., 202, 472 - 485.

[29] Khan, M.A., Ungar, I.A., Showalter A.M. (2000). Effects of Salinity on Growth, Water Relations and Ion Accumulation of The Subtropical Perennial Halophyte, Atriplex griffithii var. stocksii. Ann. Bot., 31, 2763-2774.

[30] Rahman, M., Soomro, U.A., Zahoor-ul-Haq, M., Gul, S. (2008). Effects of NaCl Salinity on Wheat (Triticum aestivum L.) Cultivars. World J. Agric. Sci., 4, 398-403.

[31] Aslan, D., Aktaş, H., Ordu, B., Zencirci, N., (2017). Evaluation of Bread and Einkorn Wheat Under in vitro Drought Stress. The J. Animal Plant Sci. 27(6), 1974-1983.

[32] Mahmoodabad, R.Z., Somarin, S.J., Khayatnezhad, M., Gholamin, R. (2001). Effect of Cold Stress on Germination and Growth of Wheat Cultivars. Adv. Environ. Biol. 5, 94-97.

[33] Foolad, M.R., Lin, G.Y. (1997). Genetic Potential for Salt Tolerance During Germination in Lycopersicon Species. Hort. Sci., 32, 296-300.

[34] Foolad, M.R., Lin, G.Y. (1998). Genetic Analysis of Low Temperature Tolerance during Germination in Tomato, Lycopersicon Esculentum, Mill. Plant Breed., 117, 171-176.

[35] Mahmoodzadeh, H., Masoudi, F.K., Besharat, H., (2013). Impact of Salt Stress on Seed Germination Indices of Five Wheat Cultivars. Ann. Biol. Res., 4, 93-96.

[36] Ali, M.B., El-Sadek, A.N. (2016). Evaluation of Drought Tolerance Indices for Wheat (Triticum aestivum L.) Under the Irrigated and Rainfed Conditions. Commun Biometry Crop Sci., 11, 77 - 89. 